\title{
Determination of the Transition-Moment Directions from Photoselection in Partially Oriented Systems*
}

\author{
A. Kawski and Z. Gryczyński \\ Luminescence Research Group, Institute of Experimental Physics, University of Gdańsk, Poland \\ and Institute of Physics, Pedagogical University, STupsk, Poland
}

Z. Naturforsch. 42 a, 808-812 (1987); received March 17, 1987

A formula is derived for the emission anisotropy $r\left(\beta, R_{\mathrm{s}}\right)$ of a polymer film as a function of the stretch ratio $R_{\mathrm{s}}$ of the film and of the angle $\beta$ between the absorbing and emitting oscillators for the case when the long axis of the molecule makes an angle $\varphi=90^{\circ}$ with the absorbing oscillator. This relation is verified, together with that obtained previously for $\varphi=0^{\circ}$, on the example of acridone and anthracene in polyvinyl alcohol. The experimental results obtained for $r\left(\beta, R_{\mathrm{s}}\right)$ as a function of $R_{\mathrm{s}}$ confirm the character of the predicted theoretical curves. The reasons for the lack of quantitative agreement is also discussed.

\section{Introduction}

The photoluminescence anisotropy of isotropic rigid or highly viscous solutions can be used to determine the angle $\beta$ between the transition moment directions in the absorption and emission [1]. This method has been reviewed by Dörr [2, 3], Albrecht [4], Liptay [5], Förster [6], and recently by Kawski [7].

We have recently considered the possibility of determining the absorption and emission transition moment directions by employing the phenomenon of photoselection in partially oriented systems, for the case when the absorption transition moment coincides with the long axis of the molecule [8]. When the excitation takes place in the long-wave absorption band, an increase in the emission anisotropy is observed as a function of the stretch ratio, $R_{\mathrm{s}}$, of a polymer film, when the angle $\beta$ between the transition moment directions in the absorption and emission does not exceed $54.7^{\circ}$ (see Fig. 2 in [8]). If the direction of the absorption transition moment in the long-wave absorption band is perpendicular to the long axis of the molecule, similarly as in acridone and anthracene, then stretching of the polyvinyl alcohol (PVA) film is accompanied by a decrease in the emission anisotropy when increasing $R_{\mathrm{s}}$.

The aim of the present paper is to investigate and explain the latter phenomenon.

* This work was carried out under contract CPBP 01.06 .

Reprint requests to Prof. Dr. Alfons Kawski, Uniwersytet Gdański, Instytut Fizyki Doświadczalnej, ul. Wita Stwosza 57, 80-952 Gdańsk, Poland.

\section{Theory}

Let us consider a prolate molecule, the directions of its long axis, $\overrightarrow{O M}$, and absorption transition moment, $\vec{A}$, making angles $\omega$ and $\omega_{1}$ with the $Z$-axis (the stretching direction of the polymer film), respectively, and being perpendicular to each other $\left(\varphi=90^{\circ}\right)$. Let $\beta$ be the angle between the directions of the absorption and emission transition moments. The electric vector, $\vec{\varepsilon}$, of the exciting light is oriented along the $Z$-axis. $J_{\|}$and $J_{\perp}$ are the components of the fluorescence intensity $\left(J=J_{\|}+2 J_{\perp}\right)$ parallel and perpendicular to $\vec{\varepsilon}$, respectively, as observed along the $X$-axis. The angles in Fig. 1 obey

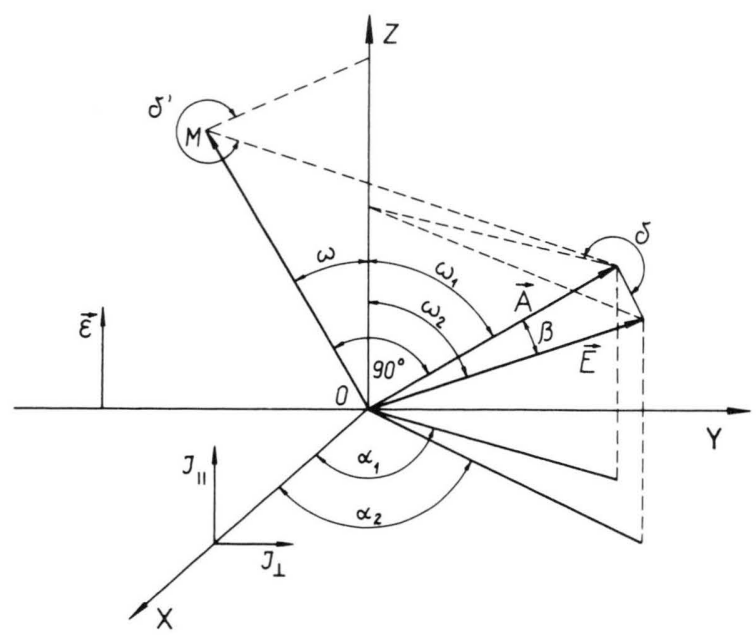

Fig. 1. Geometry of the system.

0932-0784/ 87 / 0800-0808 \$01.30/0. - Please order a reprint rather than making your own copy. 
the following relations

$\cos \omega_{2}=\cos \omega_{1} \cos \beta+\sin \omega_{1} \sin \beta \cos \delta$,

$\cos \omega_{1}=\cos \omega \cos 90^{\circ}+\sin \omega \sin 90^{\circ} \cos \delta^{\prime}$.

On squaring (1) and averaging $\cos ^{2} \omega_{2}$ over the angle $\delta\left(\langle\cos \delta\rangle=0,\left\langle\cos ^{2} \delta\right\rangle=\frac{1}{2}\right)$ we obtain [8]

$\left\langle\cos \omega_{2}\right\rangle=\left(\frac{3}{2} \cos ^{2} \omega_{1}-\frac{1}{2}\right)\left(\cos ^{2} \beta-\frac{1}{3}\right)+\frac{1}{3}$,

where $\beta$ is constant for a given transition in the molecule. Averaging over all directions $\omega_{1}$ yields the mean $\left\langle\cos ^{2} \omega_{2}\right\rangle$, which is related with the emission anisotropy $r\left(\omega_{2}\right)$ by

$$
r\left(\omega_{2}\right)=\frac{3}{2} \frac{J_{\|}}{J}-\frac{1}{2}=\frac{3}{2}\left\langle\cos ^{2} \omega_{2}\right\rangle-\frac{1}{2} .
$$

Substitution of the averaged equation (3) into (4) yields

$$
r\left(\beta, \omega_{1}\right)=\left(\frac{3}{2}\left\langle\cos ^{2} \omega_{1}\right\rangle-\frac{1}{2}\right)\left(\frac{3}{2} \cos ^{2} \beta-\frac{1}{2}\right),
$$

substituting into (5), we obtain

$$
\begin{aligned}
r(\beta, \omega)_{\varphi=90^{\circ}}= & \left(\frac{3}{2} \frac{3}{4} \frac{\int_{0}^{\pi / 2} f_{\mathrm{g}}(\omega) \sin ^{4} \omega \mathrm{d} \omega}{\int_{0}^{\pi / 2} f_{\mathrm{g}}(\omega) \sin ^{2} \omega \mathrm{d} \omega}-\frac{1}{2}\right) \\
& \cdot\left(\frac{3}{2} \cos ^{2} \beta-\frac{1}{2}\right),
\end{aligned}
$$

where $f_{\mathrm{g}}(\omega)$ is the distribution function of the long molecular axes over the angle $\omega$, given by Tanisaki's formula [9]

$f_{\mathrm{g}}(\omega)=R_{\mathrm{s}}^{2} \sin \omega\left\{1+\left(R_{\mathrm{s}}^{2}-1\right) \sin ^{2} \omega\right\}^{-3 / 2}$.

For $R_{\mathrm{s}}=1$, the expression in the first parentheses in (9) assumes a value of $2 / 5$, and changes into the known Perrin's equation [1].

For $R_{\mathrm{s}}>1$, substitution of (10) into (9) and integrating over the angle $\omega$ yields the equation

$r\left(\beta, R_{\mathrm{s}}\right)_{\varphi=90^{\circ}}=\left\{\frac{3}{2} \frac{3}{4} \frac{1+a^{2}\left(a^{2}-2\right)+\frac{1}{2} a^{2}\left(a^{2}-1\right)+a^{2}\left(a^{2}-1\right)^{1 / 2}\left(2-\frac{3}{2} a^{2}\right) \arcsin \frac{1}{a}}{1-a^{2}+a^{2}\left(a^{2}-1\right)^{1 / 2} \arcsin \frac{1}{a}}-\frac{1}{2}\right\}\left(\frac{3}{2} \cos ^{2} \beta-\frac{1}{2}\right)$,

where

$$
\left\langle\cos ^{2} \omega_{1}\right\rangle=\frac{\int_{0}^{\pi / 2} \cos ^{2} \omega_{1} \cdot f\left(\omega_{1}\right) \mathrm{d} \omega_{1}}{\int_{0}^{\pi / 2} f\left(\omega_{1}\right) \mathrm{d} \omega_{1}}
$$

and $f\left(\omega_{1}\right)$ is the direction distribution in the excited state due to the photoselection. If $f_{\mathrm{g}}\left(\omega_{1}\right)$ denotes the distribution function of the absorption oscillators in the ground state, then for the excited state we obtain

$$
f\left(\omega_{1}\right)=f_{\mathrm{g}}\left(\omega_{1}\right) \cos ^{2} \omega_{1}
$$

On substitution of (7) into (6), the following expression is obtained:

$$
\left\langle\cos ^{2} \omega_{1}\right\rangle=\frac{\int_{0}^{\pi / 2} \cos ^{4} \omega_{1} \cdot f_{\mathrm{g}}\left(\omega_{1}\right) \mathrm{d} \omega_{1}}{\int_{0}^{\pi / 2} \cos ^{2} \omega_{1} \cdot f_{\mathrm{g}}\left(\omega_{1}\right) \mathrm{d} \omega_{1}} .
$$

By employing (2) and integrating the numerator and denominator in (8) over the angle $\delta^{\prime}$, and then on where

$$
a^{2}=\frac{R_{\mathrm{s}}^{2}}{R_{\mathrm{s}}^{2}-1} \cdot *
$$

The limiting values of the emission anisotropy are:

$$
r\left(\beta, R_{\mathrm{s}}\right)_{\varphi=90^{\circ}} \rightarrow \frac{2}{5}\left(\frac{3}{2} \cos ^{2} \beta-\frac{1}{2}\right) \text { for } \quad R_{\mathrm{s}} \rightarrow 1
$$

(isotropic distribution in the ground state) with

$$
\begin{aligned}
& r\left(\beta, R_{\mathrm{s}}\right)=\frac{2}{5} \quad \text { for } \quad \beta=0, \\
& r\left(\beta, R_{\mathrm{s}}\right)=-\frac{1}{5} \text { for } \beta=90^{\circ}, \\
& r\left(\beta, R_{\mathrm{s}}\right) \rightarrow 0 \quad \text { for } \quad R_{\mathrm{s}} \rightarrow \infty \text {. }
\end{aligned}
$$

Figure 2 shows the dependence of $r\left(\beta, R_{\mathrm{s}}\right)$ on the stretch ratio $R_{\mathrm{s}}$, calculated according to (11). With increasing values of $R_{\mathrm{s}}$, for the angles $\beta$ from $0^{\circ}$ to $54.7^{\circ}$, the emission anisotropy decreases, whereas for $\beta$ ranging from $54.7^{\circ}$ to $90^{\circ}$, a growth in the emission anisotropy is predicted.

* In the previous paper [8], $a^{2}$ should have also been printed. 


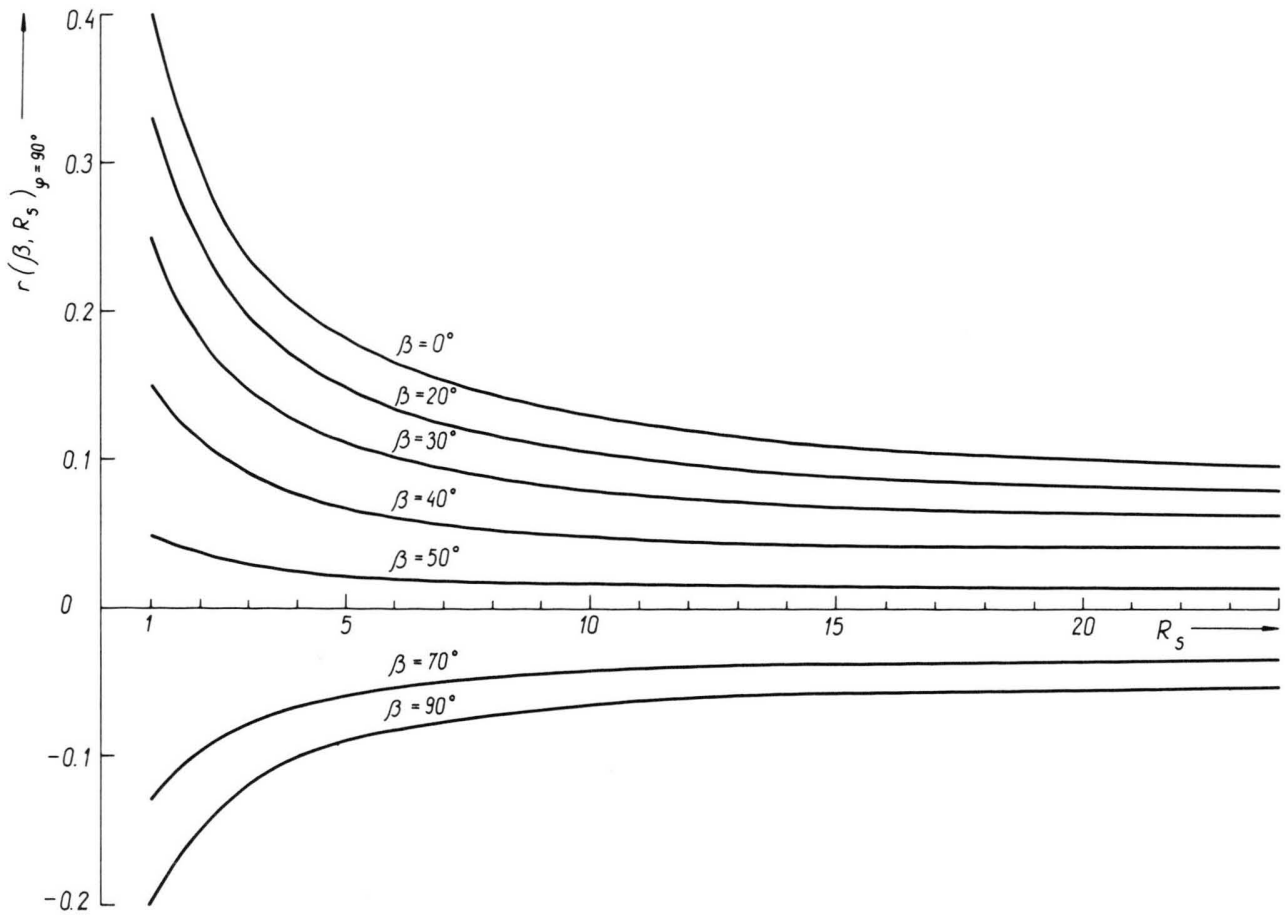

Fig. 2. Theoretical dependence of $r\left(\beta, R_{\mathrm{s}}\right)_{\varphi=90^{\circ}}$ on the stretch ratio $R_{\mathrm{s}}$ for various values of $\beta$ (11).

\section{Experimental}

\subsection{Samples and Methods}

In order to verify the validity of (11), we have selected acridone, already investigated previously [10] by measuring the linear dichroism in stretched PVA films and anthracene, which exhibits similar polarization of the transitions. Acridone displays two strong absorption bands with $\lambda_{1}^{\max }=404 \mathrm{~nm}$ and $\lambda_{2}^{\max }=259 \mathrm{~nm}[10-12]$ and mutually perpendicular polarizations. The long-wave band $\left(\lambda_{1}^{\max }=404 \mathrm{~nm}\right)$ is polarized along the $y$-axis (the shorter axis of the molecule) (Fig. 3) and is characterized by a negative value of the linear dichroism, LD. The second band $\left(\lambda_{2}^{\max }=259 \mathrm{~nm}\right)$ is polarized along the $z$-axis (the long axis of the molecule) with the LD assuming positive values.

Acridone and anthracene were introduced into the aqueous solution of PVA from a dye solution $\left(10^{-3} \mathrm{M}\right)$ in methanol. The PVA films were prepared according to the procedure described by Tanizaki [13] and stretched at the polimerization temperature of PVA $\left(\sim 80^{\circ} \mathrm{C}\right)$. The stretch degree,
$R_{\mathrm{S}}$, of the film was assumed to be the measure of the orientation $[11,13]$. The fluorescence anisotropy measurements were carried out by means of a home-built measuring apparatus [14].

\subsection{Results and Discussion}

The measured values of the emission anisotropies, $r$, for acridone and anthracene in stretched PVA films excited in the long-wave band (the angle $\varphi$ between the long axis of the molecule and the direction of the absorbing oscillator, $\vec{A}$, amounts to $90^{\circ}$ ) are shown in Figure 4. The measured values of $r$ lie close to the theoretical curve calculated according to (11) for $\beta=0^{\circ}$. The limiting values of $r$ for an isotropic solution $\left(R_{\mathrm{s}}=1\right)$ of acridone and anthracene amount to 0.285 and 0.283 , respectively. The lack of complete accordance between the experimental points and the theoretical curve can only be accounted for by the partial orientation of the relatively short molecules of acridone and anthracene, and also by other depolarizing factors. The experimental results confirm the character of 


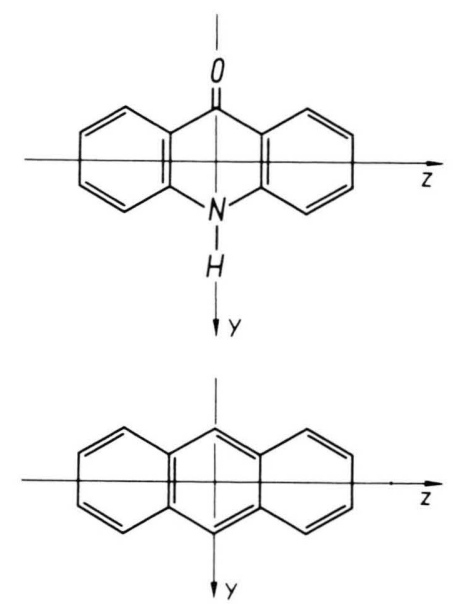

Fig. 3. Structural formulas of acridone (above) and anthracene (below).

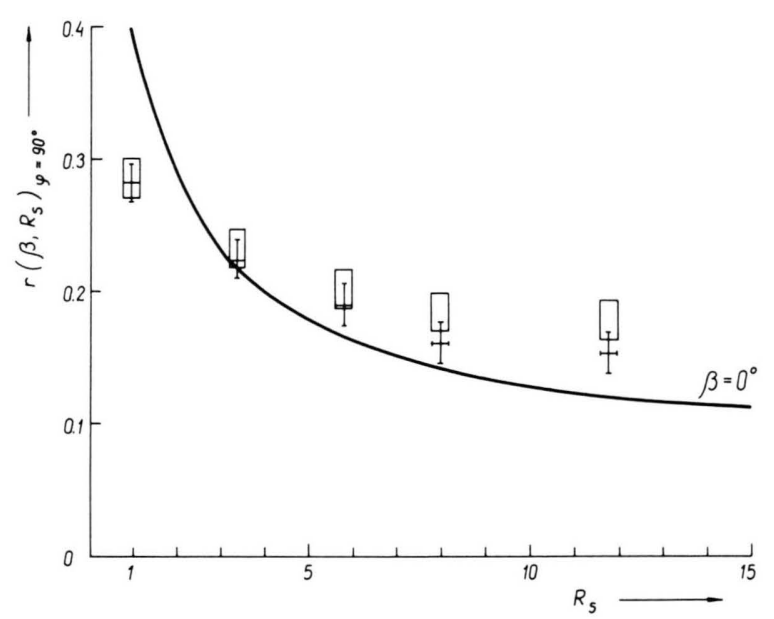

Fig. 4. Dependence of the emission anisotropy $r$ on the degree of stretching $R_{\mathrm{s}}$ for acridone $(\square)$ and anthracene $(+)$, determined by the excitation wavelengths $404 \mathrm{~nm}$ and $384 \mathrm{~nm}$, respectively. The solid line is the theoretical curve $r\left(\beta, R_{\mathrm{s}}\right)_{\varphi=90^{\circ}}$ according to (11) for $\beta=0^{\circ}$.

the dependence of $r\left(\beta, R_{\mathrm{s}}\right)$ on $R_{\mathrm{s}}$ according to (11). Therefore, it can be inferred that the absorption and emission oscillators coincide $\left(\beta \approx 0^{\circ}\right)$.

If the excitation occurs in the short-wave band, the angle $\varphi$ is close to zero since the long axis of the

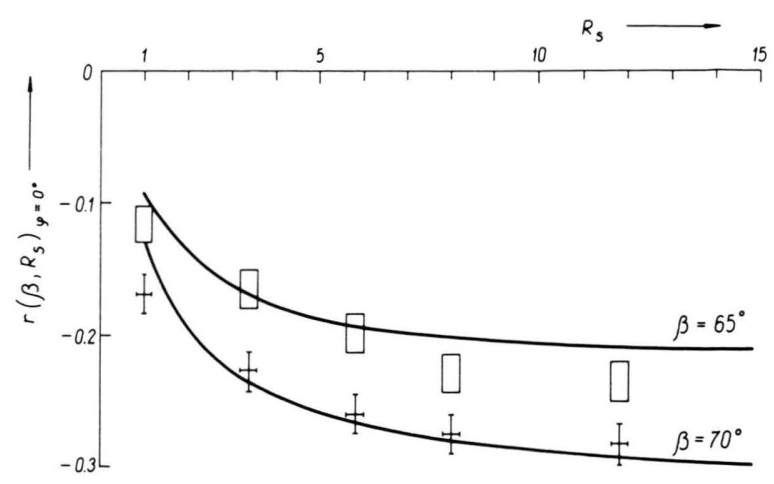

Fig. 5. Dependence of emission anisotropy $r$ on the degree of stretching $R_{\mathrm{s}}$ for acridone ( $\square$ ) and anthracene $(+)$, determined by the excitation wavelengths $259 \mathrm{~nm}$ and $255 \mathrm{~nm}$, respectively. The solid lines are the theoretical curves $r\left(\beta, R_{\mathrm{s}}\right)_{\varphi=0^{\circ}}$ according to (9) in [8] for $\beta=65^{\circ}$ and $\beta=70^{\circ}$, respectively.

molecule coincides with the direction $\vec{A}$ of the absorbing oscillator. This case is described by the formula given in the previous paper $((9)$ in [8]). The results obtained from the measurements of the emission anisotropy, $r$, are compared in Fig. 5 with the theoretical curves calculated from (9) in [8] for $r\left(\beta, R_{\mathrm{s}}\right)$. The angles $\beta$ obtained for acridone and anthracene amount to $65^{\circ}$ and $70^{\circ}$, respectively. The lack of good agreement is due to the abovementioned partial orientation of the relatively short molecules. However, the experimental values of the limiting emission anisotropy markedly differ from a theoretical value of $-0.2(-0.11$ and -0.17 for acridone and anthracene, respectively), which is due to depolarizing factors such as, for example, partial overlapping of the absorption bands with different polarization of transitions [15], torsional vibrations immediately following excitation during the thermal relaxation of the luminescent molecule $[16,17]$. Better agreement is obtained with anthracene. It can be concluded that the character of the experimental curves both for $\varphi=0^{\circ}$ and $\varphi=90^{\circ}$ confirms the predicted theoretical dependence of $r\left(\beta, R_{\mathrm{s}}\right)$ upon $R_{\mathrm{s}}$. 
Note added in proof:

Generally instead of (9) the following expression

$$
\begin{aligned}
r\left(\beta, \vartheta_{\mathrm{E}}, R_{\mathrm{s}}\right)=1-\frac{5}{4} \frac{\int_{0}^{\pi / 2} f_{\mathrm{g}}(\omega) \sin ^{4} \omega \mathrm{d} \omega}{\int_{0}^{\pi / 2} f_{\mathrm{g}}(\omega) \sin ^{2} \omega \mathrm{d} \omega} & \left(\frac{3}{2} \cos ^{2} \vartheta_{\mathrm{E}}-\frac{1}{2}\right) \\
& +\frac{1}{2} \int_{0}^{\pi / 2} \int_{\mathrm{g}}^{\pi / 2} f_{\mathrm{g}}(\omega) \sin ^{2} \omega \mathrm{d} \omega
\end{aligned}
$$

is proper, where $\vartheta_{E}$ denote the angle between the directions of the long axis $\overrightarrow{O M}$ and the emission transition moment $\vec{E}$ (see Figure 1). When the plane in which the vectors $\overrightarrow{O M}$ and $\vec{A}$ are situated, create an angle $\pi / 4$ with the plane in which the vectors $\vec{A}$ and $\vec{E}$ lie, then (13) assumes the simple form (9) or (11) describing the theoretical curves in Figure 2. For $\beta=0$ equations (11) and (13) are presented by the same curve (Figure 4). By employing (10) and integrating the numerator and denominator in (13) over the angle $\omega$, we obtain

$$
\frac{\int_{0}^{\pi / 2} f_{\mathrm{g}}(\omega) \sin ^{4} \omega \mathrm{d} \omega}{\int_{0}^{\pi / 2} f_{\mathrm{g}}(\omega) \sin ^{2} \omega \mathrm{d} \omega}=\frac{3}{4} \frac{\left.1+a^{2}\left(a^{2}-2\right)+\frac{1}{2} a^{2}\left(a^{2}-1\right)+a^{2}-1\right)^{1 / 2}\left(2-\frac{3}{2} a^{2}\right) \arcsin (1 / a)}{1-a^{2}+a^{2}\left(a^{2}-1\right)^{1 / 2} \arcsin (1 / a)},
$$

where $a^{2}$ is given by (12).

We are indebted to Doc. Dr. habil. T. Marszałek for calling our attention that the Eq. (13) can be expressed in this generalized way.

[1] F. Perrin, Ann. Physique 12, 169 (1929).

[2] F. Dörr and H. Held, Angew. Chem. 72, 287 (1960).

[3] F. Dörr, Angew. Chem. (Intern. Ed. in English) 5, 478 (1966).

[4] A. C. Albrecht, J. Molec. Spectr. 6, 84 (1961).

[5] W. Liptay, in: Modern Quantum Chemistry, Part 3, Acad. Press Inc., New York 1965, p. 81.

[6] Th. Förster, Pure and Appl. Chem. 11, 287 (1965).

[7] A. Kawski, Proceedings of the Fourth Symposium on Optical Spectroscopy, Teubner-Texte zur Physik, Leipzig 1986.

[8] A. Kawski and Z. Gryczyński, Z. Naturforsch. 41 a, 1195 (1986).

[9] Y. Tanizaki, Bull. Chem. Soc. Japan 38, 1798 (1965).
[10] Z. Gryczyński and A. Kawski, Acta Phys. Polon. A70, 105 (1986).

[11] Y. Matsuoka and B. Nordén, Chem. Phys. Lett. 85, 302 (1982).

[12] H. Inoue, T. Hoshi, and J. Yoshino, Bull. Chem. Soc. Japan 45, 2653 (1972).

[13] Y. Tanizaki, Bull. Chem. Soc. Japan 32, 75 (1959).

[14] A. Kawski, Z. Kojro, and A. Kubicki, Z. Naturforsch. 40 a, 313 (1985).

[15] A. Kawski, Acta Phys. Polon. 35, 863 (1968).

[16] A. Jabloński, Acta Phys. Polon. 10,33, 193 (1950).

[17] A. Kawski, A. Kubicki, I. Weyna, and I. Janić, Z. Naturforsch. 40 a, 559 (1985). 